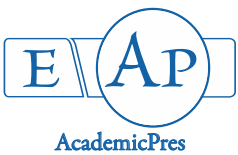

Konyeme TE et al. (2021)

Notulae Scientia Biologicae

Volume 13, Issue 2, Article number 10829

DOI: $10.15835 / \mathrm{nsb} 13210829$

Research Article

\title{
Intraspecific diversity of Vigna subterranea accessions obtained from multinational sources
}

\author{
Thelma E. KONYEME ${ }^{1 *}$, Josephine U. AGOGBUA ${ }^{1}$, \\ Daniel B. ADEWALE ${ }^{2}$, Chinedum OGAZIE ${ }^{1}$
}
${ }^{1}$ University of Port Harcourt, Department of Plant Science and Biotechnology, Rivers State, Nigeria; konyeme.thelma@gmail.com (*corresponding author); josephine.agogbua@uniport.edu.ng; chinedum.ogazie@uniport.edu.ng
${ }^{2}$ Federal University Oye-Ekiti, Department of Crop Science and Horticulture, Ekiti State, Nigeria; d.adewale@gmail.com

\begin{abstract}
Bambara groundnut is a legume with balanced meal. The present study investigated agro-morphological diversity of 61 Bambara groundnut accessions whose passport data were from 21 countries. The Genetic Resources Centre (GRC), International Institute of Tropical Agriculture (IITA), Ibadan, Nigeria provided the 61 accessions. The accessions were established in row plots of ten plants on the field at the Centre for Ecological studies, University of Port Harcourt, Nigeria. Seventeen quantitative and two qualitative traits were recorded from the sampling units for each accession. Descriptive and multivariate statistical analysis were employed on the $61 \times 19$ matrix mean data. Significant variation exists among the 61 accessions for the 19 descriptors. Mean genetic similarity among the 61 accessions was 0.78 , the least $(0.48)$ similarity was between TVSu1750 and TVSu250 and the highest (0.92) was between TVSu391 and TVSu415. TVSu1843 flowered earliest at 35 days. The 61 accessions were grouped into three main clusters. TVSu1964 and two accessions from Malawi (TVSu1748 and TVSu1750) in cluster III had high grain yield. Accessions in cluster II were significant for both vigour and grain yield. Striking uniformity and diversity existed among accessions from the same country. Genetic improvement in vigour and grain yield of Bambara groundnut is possible within each country.
\end{abstract}

Keywords: Bambara groundnut; biplot; dendogram; intraspecific diversity; multinational; principal components

\section{Introduction}

Bambara groundnut (Vigna subterranean [L.] Verdc. formerly known as Voandzie subterranea (L.)) is an herbaceous, annual, autogamous (Heller et al., 1997), underutilized food legume (Azam-Ali et al., 2001). Among the notable African legumes, Bambara groundnut comes after cowpea (Vigna unguiculata) and groundnut (Arachis hypogea) (Ntundu et al., 2004; 2006) in terms of production and importance. West African countries such as Burkina Faso, Cameroon, Mali and Niger hosts its nativity Heller et al. (1997) and leads global production (FAO, 2015). Its cultivation and utilization outside Africa are rare (Hillock et al., 2011) as food, feed and soil ameliorator. Its production is widely distributed throughout the semi-arid zone of

Received: 03 Oct 2020. Received in revised form: 31 Mar 2021. Accepted: 29 Apr 2021. Published online: 05 May 2021. From Volume 13, Issue 1, 2021, Notulae Scientia Biologicae journal uses article numbers in place of the traditional method of continuous pagination through the volume. The journal will continue to appear quarterly, as before, with four annual numbers. 
sub-Saharan Africa and yields well under conditions which are too arid for groundnut, maize and even sorghum (Thottappilly and Rossel, 1997). It is a crop of significant relevance for food, nutrition, edaphic and climatic security for Africa.

Ntundu et al. (2004) identified the centre of diversity for Bambara groundnut to be 'Bambara' a place near Timbuktu in present day republic of Mali, West Africa (The crop is widely cultivated in sub-Saharan Africa where it has adapted to various agro-ecosystems according to Bonny et al. (2019). Benin, Burkina Faso, Cote d'Ivoire, Gambia, Ghana, Guinea, Mali, Nigeria, Niger, Senegal and Togo according to Goli (1997) are some of the major producing countries in west Africa. Uguru and Ezeh (1997) described it to morphologically resemble groundnut (Arachis hypogea (L.)). It measures about $0.30-0.35 \mathrm{~m}$ high, smallish in nature with trifoliate compound-leaf. According to Tweneboah (2000), Bambara groundnut exhibits two morphotypes which are: erect and prostrate.

Although it is not a crop with global fame, but the national production and consumption statistics (Baudoin and Mergeai, 2001) revealed that the seed is an essential part in the diet of a lot of people in West Africa. The meal and medicinal qualities of the crop is vast across cultures, nationals and regions. In the eastern part of Nigeria, the seeds are made into pudding called Okpa (Okpuzo et al., 2009). The Zybo tribe in Nigeria uses it for the treatment of venereal diseases (Brink et al., 2006). In Zambia, it is a component for bread making (Brough et al., 1993). In Zimbabwe, it is either eaten fresh or dried and stored to be relished later (Hillocks et al., 2001). In east Africa, it is roasted, then pulverized and used for making soup. (Linnemann, 1990). Livestock feeds which is rich in phosphorus and protein is obtained from the leaves (Drabo et al., 1995) etc. Bambara groundnut is the only legume whose seeds are referred to as complete food because of the balanced proportioned content of fat, carbohydrate and protein (Poulter and Caygil, 1980).

Despite the vast knowledge on the crop's nutrition and cultural productive utilities, its genetic resources still exist as mere landraces and at most, conserved accessions. Valombola et al. (2019) report on the Namibian accessions hinted that the crop had neither received a pre-breeding nor a breeding programme, they are informally classified and named according to areas of production and market all because it is an underutilized species. Ntundu et al. (2004) equally noted that breeding and improvement programme of the crop is scarce. However, there are some reports (Goli et al., 1997; Pasquet et al., 1999, Ntudu et al., 2004; 2006; Unigwe et al., 2016; Valombola et al., 2019; Bonny et al., 2019) about the agro-morphological diversity of the crop, identifying it as a crop with wide intra-population diversity but narrow intra-specific variability.

GRC, IITA, Ibadan, Nigeria hosts high quantity of the genetic resources of Bambara groundnut from different nations of the world especially Africa. Sixty-one accessions from different nations in the regions of Africa and outside Africa were obtained from GRC for the present study with the aim of assessing intraspecific variability among them based on morphological markers and to ascertain possible uniqueness among accessions from the same national sources. This in turn is hoped to inform plant breeders of the status and availability of genetic resources to work with in their national or other national domains in Africa.

\section{Materials and Methods}

Sixty-one (61) Bambara groundnut accessions from 21 nations sourced from GRC, IITA, Ibadan, Nigeria were used for the study. See the list of accessions with the corresponding countries of origin in Table 1. The treatment was not replicated on the field following the method of Ofori et al. (2006) and Morris (2008) because the aim of the experiment was characterization assessment of the 61 accessions. The experiment was conducted between May to September 2015 at the centre for Ecological studies, Department of Plant Science and Biotechnology, University of Port Harcourt, Rivers State, Nigeria. Port Harcourt is in a humid forest zone of Nigeria within latitude $4^{\circ} \mathrm{N}$ and $5^{\circ} \mathrm{N}$ and longitude $6^{\circ} \mathrm{E}$ and $7^{\circ} \mathrm{E}$.

With the sole aim of characterization and diversity assessment, an un-replicated experiment was laid out following the method of (Ofori et al., 2006; Morris, 2008). Marked area was cleared, ploughed, harrowed and 
prepared to mini mounds. Ten mini mounds were the plot size for each treatment. Planting of two seeds at a depth of $2 \mathrm{~cm}$ was done on each hill at $50 \mathrm{~cm}$ by $40 \mathrm{~cm}$ spacing. Thinning to one plant per stand was done at two weeks after planting. Weeds was manually controlled as at when due during the experiment.

Nineteen (17 quantitative and two qualitative) data were collected on each of the accessions following the Bambara groundnut descriptor (IPGRI et al., 2000). Collected data on the 61 accessions were subjected to: descriptive statistics, genetic distance, principal component and cluster analysis (Ward, 1963). Moreover, mean for each parameter for all accession from each country were generated. The 21 (countries) $\times 19$ (variables) matrix table were subjected to Gower genetic distance (Gower, 1971) and then to principal component analysis to obtain component scores for the axes. Scores of the first three axes was used to generate a tri-dimensional graph to display diversity among the 21 countries based on the 19 traits. All the analysis above were conducted using SAS (Version 9.4, 2011). To assess relationship of the quantitative trait with the 61 accessions and the 21 countries, the Statistical Tool for Agricultural Research (STAR, 2014) software were employed to singly generate biplot graphs with principal component axes 1 and 2 .

Table 1. List of Bambara groundnut accessions and their corresponding native country

\begin{tabular}{|c|c|c|c|}
\hline $\mathrm{S} / \mathrm{N}$ & List of accessions & $\begin{array}{c}\text { No. of } \\
\text { accessions }\end{array}$ & Countries \\
\hline 1 & TVSu 189, TVSu 1092 & 2 & Benin Republic \\
\hline 2 & TVSu 316, TVSu 1171 & 2 & Burkina Faso \\
\hline 3 & TVSu 415, TVSu 456, TVSu 473, TVSu 486 & 4 & Cameroon \\
\hline 4 & TVSu 1277, TVSu 1289, TVSu 1290, TVSu 1296 & 4 & Central Africa \\
\hline 5 & TVSu 1986, TVSu 1988, TVSu 1989 & 3 & DR Congo \\
\hline 6 & TVSu 115, TVSu 118 & 2 & Cote d'Ivoire \\
\hline 7 & TVSu 247, TVSu 248, TVSu 250 & 3 & Gambia \\
\hline 8 & TVSu 146, TVSu 163, TVSu 168 & 3 & Ghana \\
\hline 9 & TVSu 1146, TVSu 1150 & 2 & Guinea \\
\hline 10 & TVSu 1271, TVSu 1272, TVSu 819 & 3 & Madagascar \\
\hline 11 & TVSu 1748, TVSu 1750, TVSu 1753, TVSu 1756 & 4 & Malawi \\
\hline 12 & TVSu 89, TVSu 91, TVSu 100, TVSu 101 & 4 & Mali \\
\hline 13 & TVSu 1843, TVSu 1844 & 2 & Niger \\
\hline 14 & TVSu 30, TVSu 173, TVSu 182, TVSu 258, TVSu 278 & 5 & Nigeria \\
\hline 15 & TVSu 1647, TVSu 1649 & 2 & Senegal \\
\hline 16 & TVSu 391 & 1 & Sudan \\
\hline 17 & TVSu 1964, TVSu 1972 & 2 & Switzerland \\
\hline 18 & TVSu 183, TVSu 369, TVSu 374, TVSu 381 & 4 & Tanzania \\
\hline 19 & TVSu 1379 & 1 & United Kingdom \\
\hline 20 & TVSu 681, TVSu 682, TVSu 684, TVSu 696 & 4 & Zambia \\
\hline 21 & TVSu 1002, TVSu 1027, TVSu 1066, TVSu 1081 & 4 & Zimbabwe \\
\hline
\end{tabular}

\section{Results}

The mean, minimum and maximum values for 17 quantitative out of the 19 traits employed for the diversity analysis of the 61 Bambara groundnut accessions is presented in Table 2. Moreover, the coded scores for the two qualitative traits are present within the table. The minimum to the maximum values for each variable and other descriptive statistics shows existing variation within the 61 Bambara groundnut genetic resources (Table 2). The five principal component axes shown in Table 3 has $>1.0$ eigenvalue. The highest (27.4\%) variance proportion was in PC1 and the least (7.2\%) occurred in PC5. The total variance from the five PC axes was $65.4 \%$ (Table 3 ). The proportional contribution of each variables to the total variance is shown as eigenvector loadings, the prominent variables with eigenvector $\geq 0.3$ in PC1 were: Canopy8WAP, pods/plant, 
seeds/plant and pods with one seed (Table 3). List of variables with significant eigenvector loadings in PC2 were: Petiole length, pods/plant, seeds/plant and pods with two seeds and seed length, in PC3, notable significant variables were: 100 seed weight, leaf width, canopy at 6 and 8 weeks, pod length and seed coat colour (Table 3). Days to flowering, plant height at 6 weeks, leaf length, seedling percentage, and seed width were significantly (eigenvector $\geq 0.3$ ) prominent in PC4 and PC5 (Table 3).

Table 2. Descriptive statistics of the nineteen descriptors employed for diversity analysis of the sixty-one accessions of Bambara groundnut

\begin{tabular}{|c|c|c|c|}
\hline Variable & Mean \pm SE & Minimum & Maximum \\
\hline \multicolumn{4}{|c|}{ Quantitative variables } \\
\hline DOF (days) & $42.01 \pm 0.54$ & 30.75 & 53.25 \\
\hline Wt100 (g) & $58.51 \pm 1.54$ & 36.81 & $89.4 \mathrm{~g}$ \\
\hline PH6WAP $(\mathrm{cm})$ & $25.35 \pm 0.64$ & 17.13 & 50.35 \\
\hline PH8WAP $(\mathrm{cm})$ & $25.86 \pm 0.69$ & 17.35 & 48.55 \\
\hline Leaf length $(\mathrm{mm})$ & $4.78 \pm 0.11$ & 2.54 & 7.04 \\
\hline Leaf width $(\mathrm{mm})$ & $2.40 \pm 0.15$ & 1.26 & 5.94 \\
\hline Petiole length $(\mathrm{cm})$ & $13.68 \pm 0.31$ & 9.21 & 19.38 \\
\hline Canopy6WAP $(\mathrm{cm})$ & $51.43 \pm 1.38$ & 25.00 & 70.00 \\
\hline Canopy8WAP $(\mathrm{cm})$ & $59.71 \pm 1.44$ & 30.00 & 80.00 \\
\hline Pods/Plant & $27.15 \pm 4.35$ & 1.00 & 148.00 \\
\hline Seed/Plant & $31.03 \pm 5.01$ & 11.00 & 177.00 \\
\hline Pods with 1seed & $23.52 \pm 3.76$ & 15.00 & 132.00 \\
\hline Pods with 2 seeds & $3.78 \pm 0.80$ & 11.00 & 33.00 \\
\hline Pod length $(\mathrm{mm})$ & $1.55 \pm 0.04$ & 0.96 & 2.28 \\
\hline Shelling percent (\%) & $32.99 \pm 1.40$ & 13.64 & 86.05 \\
\hline Seed length $(\mathrm{mm})$ & $1.02 \pm 0.02$ & 0.70 & 1.30 \\
\hline Seed width & $0.80 \pm 0.01$ & 0.58 & 1.03 \\
\hline \multicolumn{4}{|c|}{ Qualitative variables } \\
\hline Hilum colour & & 1 & 5 \\
\hline Seed coat colour & & 1 & 7 \\
\hline
\end{tabular}

DOF $=$ Days to flowering, Wt100 = weight of 100 seeds, PH6WAP $=$ Plant height at 6 weeks after planting, PH8WAP $=$ Plant height at 8 weeks after planting, Canopy6WAP $=$ Canopy at 6 weeks after planting, Canopy8WAP $=$ Canopy at 8 weeks after planting, Shelling $\%=$ shelling percentage

Hilum colour: 1 = White eye, 2 = White eye with purple butterfly hallow, $3=$ White eye with black hallow, $4=$ White eye grey hallow and $5=$ White eye with thin brown hallow

Seed coat colour: $1=$ Cream, $2=$ Brown, $3=$ Purple, $4=$ Wine Red, $5=$ Grey, $6=$ Mottled and $7=$ Blue black

The 61 accessions scattered spatially in the four quadrants while the 17 quantitative variables appeared in quadrants 1, 3 and 4 (Figure 1). In quadrant 1, the 11 accessions had the best performance for hundred seed weight, days to flowering and shelling percentage, strong positive correlation existed between 100 seed weight and days to flowering (Figure 1). Highly significant positive correlation was observed between pods/plant, seeds/plant and one seed/pod in the third quadrant (Figure 1) because of the acute angle between the projections of the three traits. The eight accessions within the third quadrant were outstanding in their high performances for the yield related traits (Figure 1). The remaining 10 variables (which were mostly vegetative and yield related traits) and about twenty Bambara groundnut accessions featured in quadrant 4 (Figure 1). Among the twenty accessions, TVSu 1271 had the longest seed length while TVSu1756 and TVSu696 had the longest leaf length. Within the fourth quadrant, correlation between leaf width and canopy at 6 weeks after planting, then plant height at 6 and 8 weeks after planting were positive and highly significant (Figure 1). 
Table 3. Eigenvalues, variance components of five PC axes and the different proportional contributions of the 19 traits as Eigenvectors loadings in the five PC axes

\begin{tabular}{|l|c|c|c|c|c|}
\hline & PC1 & PC2 & PC3 & PC4 & PC5 \\
\hline Eigenvalue & 5.197 & 2.750 & 1.646 & 1.465 & 1.360 \\
\hline Proportion & 0.274 & 0.145 & 0.087 & 0.077 & 0.072 \\
\hline Cumulative & 0.274 & 0.418 & 0.505 & 0.582 & 0.654 \\
\hline \multicolumn{5}{|c|}{ Eigenvectors } \\
\hline DOF O (days) & -0.087 & 0.071 & 0.153 & -0.069 & 0.571 \\
\hline Wt100 (g) & -0.124 & 0.152 & 0.409 & 0.103 & -0.369 \\
\hline PH6WAP (cm) & 0.229 & 0.255 & 0.038 & 0.013 & 0.310 \\
\hline PH8WAP (cm) & 0.250 & 0.275 & 0.103 & -0.097 & 0.197 \\
\hline Leaf length (mm) & 0.206 & 0.040 & -0.178 & 0.455 & 0.009 \\
\hline Leaf width (mm) & 0.150 & 0.106 & -0.445 & 0.307 & 0.192 \\
\hline Petiole length (cm) & 0.264 & 0.325 & -0.122 & 0.127 & 0.177 \\
\hline Canopy6WAP (cm) & 0.228 & 0.152 & 0.374 & 0.158 & -0.114 \\
\hline Canopy8WAP (cm) & 0.309 & 0.135 & 0.373 & -0.016 & 0.037 \\
\hline Pods/Plant & 0.358 & -0.317 & 0.068 & -0.082 & 0.028 \\
\hline Seed/Plant & 0.356 & -0.330 & 0.058 & -0.083 & 0.005 \\
\hline Pods with 1seed & 0.353 & -0.298 & 0.083 & -0.087 & 0.062 \\
\hline Pods with 2 seeds & 0.287 & -0.328 & -0.016 & -0.058 & -0.140 \\
\hline Pod length (mm) & 0.150 & 0.146 & -0.330 & -0.164 & -0.241 \\
\hline Shelling percent (\%) & -0.068 & 0.182 & 0.138 & -0.532 & 0.184 \\
\hline Seed length (mm) & 0.193 & 0.360 & -0.058 & -0.116 & -0.324 \\
\hline Seed width & 0.206 & 0.270 & -0.120 & -0.168 & -0.314 \\
\hline Hilum colour & -0.078 & -0.096 & -0.154 & -0.137 & -0.026 \\
\hline Seed coat colour & -0.073 & -0.009 & 0.300 & 0.487 & -0.019 \\
\hline
\end{tabular}

DOF $=$ Days to flowering, Wt100 = weight of 100 seeds, PH6WAP = Plant height at 6 weeks after planting, PH8WAP = Plant height at 8 weeks after planting, Canopy6WAP = Canopy at 6 weeks after planting, Canopy8WAP $=$ Canopy at 8 weeks after planting, Shelling $\%=$ shelling percentage

The cluster was generated from the Gower distance showing similarity coefficient among pairs of accessions (Table not shown). Similarity among the 61 accessions ranged from 0.483 (the least) between TVSu1750 (Malawi) and TVSu250 (Gambia) to 0.927 between TVSu415 (Cameroon) and TVSu391 (Sudan). However, the mean similarity among the 61 accession was 0.784 . In Figure 2, at the inflection point 0.00 , the 61 accessions were unique taxa. At the 0.05 similarity coefficient had grouped the 61 accessions into five clusters of 19, 13, 8, 18 and 3 members. At 0.10 , three groups of 19,39 and 3 members subsisted, but at the similarity coefficient of 0.15 , the 61 accessions had become a single cluster (Figure 2). The first two accessions to merge were Tvsu391 and Tvsu 415 at 0.0016 (Figure 2). The 19 accessions grouped in cluster I were from: Gambia, Cote d'Ivoire, UK, Cameroon, Mali, Burkina Faso, Benin Republic, Nigeria, Malawi, Guinea, Senegal and Niger. The 39 accessions in cluster II were from: Zimbabwe, Zambia, Niger, Mali, Tanzania, Nigeria, Madagascar, Switzerland, DR Congo, Central Africa, Guinea, Cote d'Ivoire, Ghana, Senegal, Cameroon and Sudan. Two (TVSu1743 and TVSu1750) of the three accessions in cluster III were from Malawi and TVSu1964 was from Switzerland (Figure 2). 


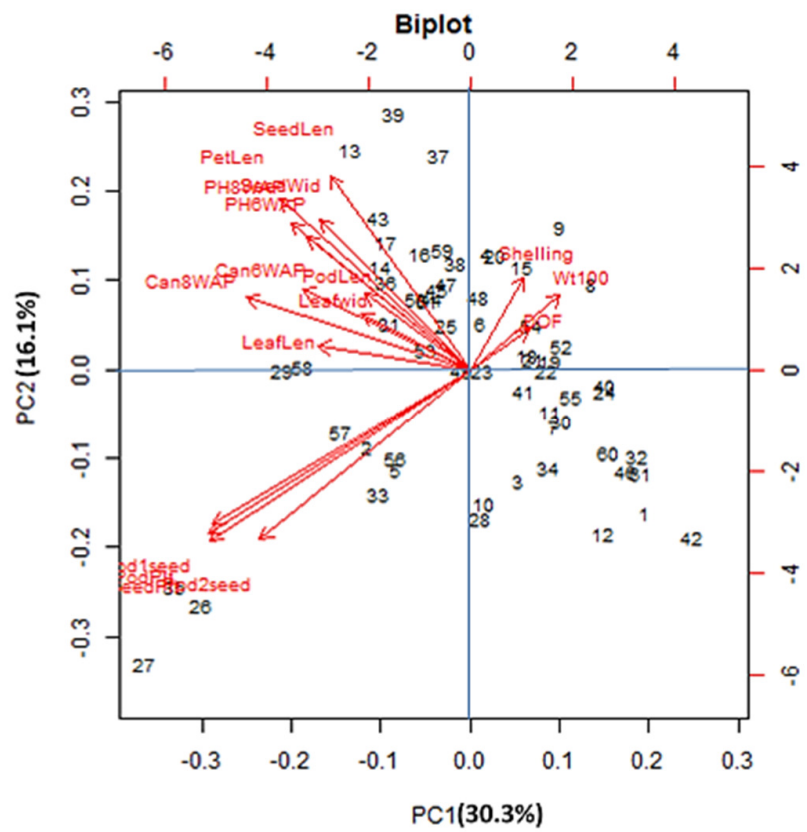

Figure 1. Biplot display of the relationship between the sixty-one Bambara groundnut accessions and seventeen quantitative variables

1 -TVSu100, 2-TVSu 1002, 3-TVSu 100, 4-TVSu 1027, 5-TVSu 1066, 6-TVSu 1081, 7-TVSu 1092, 8-TVSu 1146, 9-TVSu 115, 10-TVSu 1150, 11-TVSu 1171, 12-TVSu 118, 13-TVSu 1271, 14-TVSu 1272, 15-TVSu 1277, 16TVSu 1289, 17-TVSu 1290, 18-TVSu 1296, 19-TVSu 1379, 20-TVSu 146, 21-TVSu 163, 22-TVSu 1647, 23-TVSu 1649, 24-TVSu 168, 25-TVSu 173, 26-TVSu 1748, 27-TVSu 1750, 28-TVSu 1753, 29-TVSu 1756, 30-TVSu 182, 31-TVSu 183, 32-TVSu 1843, 33-TVSu 1844, 34-TVSu 189, 35-TVSu 1964, 36-TVSu 1972, 37-TVSu 1986, 38 TVSu 1988, 39-TVSu 1989, 40-TVSu 247, 41-TVSu 248, 42-TVSu 250, 43-TVSu 258, 44-TVSu 278, 45-TVSu 30, 46-TVSu 316, 47-TVSu 369, 48-TVSu 374, 49-TVSu 381, 50-TVSu 391, 51-TVSu 415, 52-TVSu 456, 53-TVSu 475, 54-TVSu 486, 55-TVSu 681, 56-TVSu 682,57-TVSu 684,58-TVSu 696, 59-TVSu 819, 60-TVSu 89, 61TVSu91

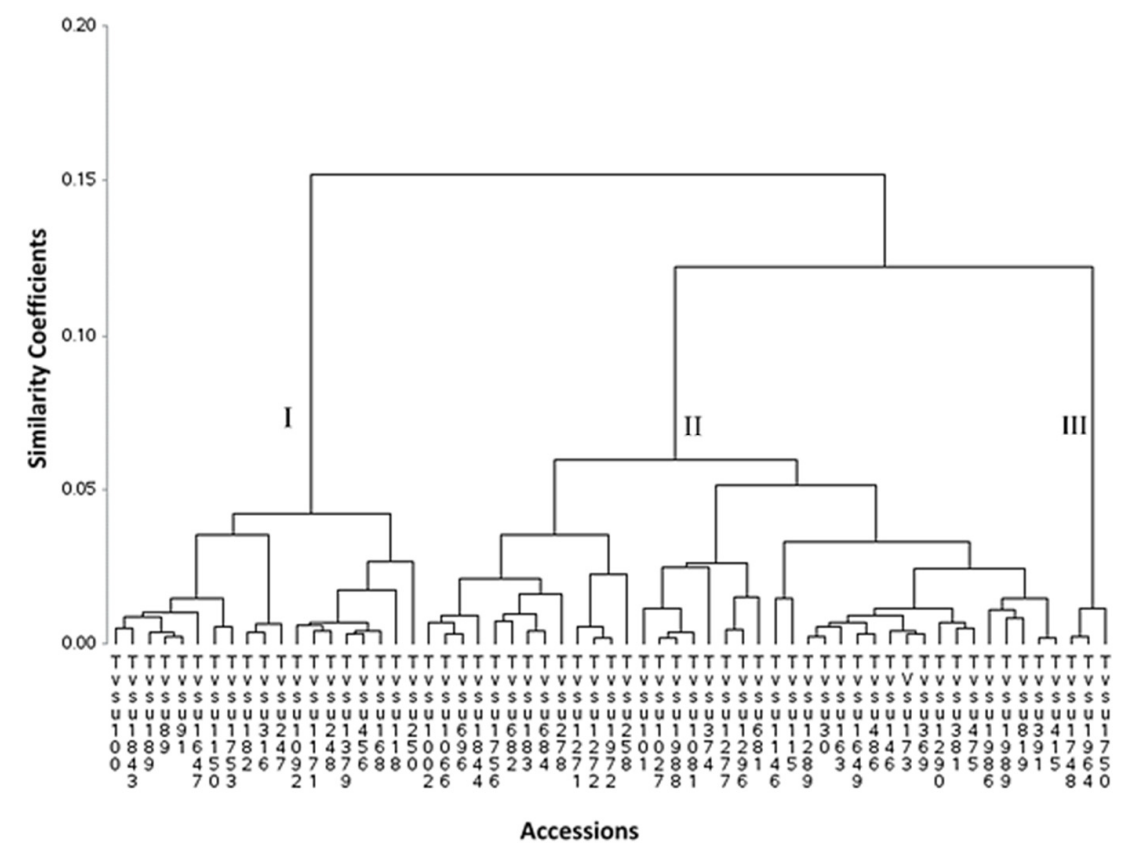

Figure 2. Dendrogram showing distinctive groupings of the 61 Bambara groundnut accession into clusters 
Table 4 provided some details on the mean performances and measures of variability of the 17 quantitative traits and the proportions of the different ordinal levels of the two quantitative traits within each of the three clusters in Figure 2. The three accessions in cluster III had the highest mean performances for ten of 17 quantitative traits, accessions in cluster II were most significant for six traits while the 19 accessions in cluster I had the highest weight for 100 seed weight (Table 4). The accessions in cluster III which were from Mali and Swaziland had two seeds in a pod, with the weight of a hundred seed of $47 \mathrm{~g}$ compared with 100 seed weight of $60 \mathrm{~g}$ in cluster I (Table 4). Another important quality in the accessions in cluster III was that it had the least shelling percentage of $28 \%$ (Table 4). Seed coat and hilum colours varied in proportion among different accessions captured within each cluster. The respective predominant seed coat colour in clusters I, II and III was cream (32\%), cream (44\%) and brown (67\%), and the commonest hilum colour for the three clusters was white (Table 4).

Table 4. Measures of average, variability and proportion of the quantitative and qualitative traits within each of the three clusters

\begin{tabular}{|c|c|c|c|c|c|c|}
\hline \multirow{2}{*}{ Variables } & \multicolumn{2}{|c|}{ Cluster I } & \multicolumn{2}{|c|}{ Cluster II } & \multicolumn{2}{|c|}{ Cluster III } \\
\hline & Mean & $\mathrm{CV}(\%)$ & Mean & CV $(\%)$ & Mean & CV (\%) \\
\hline DOF (days) & 42.09 & 9.54 & 41.92 & 10.54 & 42.67 & 9.10 \\
\hline Wt100 $(\mathrm{g})$ & 59.46 & 18.59 & 58.92 & 21.25 & 47.26 & 19.19 \\
\hline PH6WAP $(\mathrm{cm})$ & 21.21 & 11.52 & 27.20 & 17.95 & 27.48 & 3.97 \\
\hline PH8WAP $(\mathrm{cm})$ & 20.46 & 11.25 & 28.28 & 16.54 & 28.67 & 10.28 \\
\hline Leaf length (mm) & 4.29 & 16.46 & 4.97 & 17.30 & 5.41 & 18.32 \\
\hline Leaf width $(\mathrm{mm})$ & 1.90 & 38.74 & 2.65 & 48.61 & 2.42 & 12.07 \\
\hline Petiole length $(\mathrm{cm})$ & 11.20 & 10.69 & 14.82 & 13.85 & 14.53 & 6.22 \\
\hline Can6WAP $(\mathrm{cm})$ & 46.32 & 22.14 & 53.14 & 19.72 & 61.67 & 4.68 \\
\hline Can8WAP $(\mathrm{cm})$ & 51.05 & 18.35 & 62.63 & 14.97 & 76.67 & 3.77 \\
\hline Pods/Plant & 11.74 & 123.46 & 25.49 & 86.20 & 146.33 & 1.42 \\
\hline Seed/Plant & 13.21 & 123.58 & 29.18 & 86.84 & 168.00 & 4.65 \\
\hline Pod with 1 seed & 10.26 & 124.77 & 22.21 & 87.79 & 124.67 & 9.50 \\
\hline Pod with 2 seeds & 1.47 & 155.67 & 3.54 & 143.06 & 21.67 & 45.30 \\
\hline Pod length $(\mathrm{mm})$ & 1.40 & 30.03 & 1.62 & 15.24 & 1.54 & 8.11 \\
\hline Shelling percent (\%) & 31.26 & 24.36 & 34.19 & 36.48 & 28.31 & 12.63 \\
\hline Seed length $(\mathrm{mm})$ & 0.93 & 10.50 & 1.07 & 9.74 & 1.03 & 11.20 \\
\hline Seed width & 0.75 & 11.84 & 0.84 & 11.48 & 0.82 & 4.95 \\
\hline \multicolumn{7}{|c|}{ Quantitative variables } \\
\hline \multirow{2}{*}{ Cluster } & \multicolumn{6}{|c|}{ Seed coat colour } \\
\hline & Cream & Brown & Purple & Wine red & Mottled & Blue-black \\
\hline Cluster I & $31.6 \%$ & $26.3 \%$ & $5.3 \%$ & $10.5 \%$ & $15.8 \%$ & $10.5 \%$ \\
\hline Cluster II & $43.6 \%$ & $30.8 \%$ & $2.6 \%$ & $12.8 \%$ & $10.2 \%$ & Nil \\
\hline \multirow[t]{3}{*}{ Cluster III } & $33.3 \%$ & $66.7 \%$ & Nil & Nil & Nil & Nil \\
\hline & \multicolumn{6}{|c|}{ Hilum colour } \\
\hline & 1 & 2 & 3 & 4 & 5 & \\
\hline Cluster I & $68.4 \%$ & $15.8 \%$ & Nil & Nil & $15.8 \%$ & \\
\hline Cluster II & $66.7 \%$ & $28.2 \%$ & $2.5 \%$ & $2.6 \%$ & Nil & \\
\hline Cluster III & $66.7 \%$ & $33.3 \%$ & Nil & Nil & Nil & \\
\hline
\end{tabular}

† DOF $=$ Days to flowering, Wt100 $=$ weight of 100 seeds, LeafLen $=$ Leave length, Leafwid $=$ Leave width, Petlen $=$ Petiole length, Podplt $=$ Number of pods per plant, Seedplt $=$ Number of seeds per plant, PodLen $=$ Pod length, PH6WAP = Plant height at 6 weeks after planting, PH8WAP = Plant height at 8 weeks after planting, Can6WAP = Canopy at 6 weeks after planting, Can8WAP $=$ Canopy at 8 weeks after planting, Shelling $=$ shelling percentage, SeedLen $=$ Seed length, SeedWid $=$ Seed width. $†$ Hilum colour codes: $1=$ White eye, $2=$ White eye with purple butterfly hallow, $3=$ White eye with black hallow, $4=$ White eye with grey hallow and $5=$ White eye with thin brown hallow 
Table 5 contained the coefficient of variations (percentage) value for the 17 quantitative traits for the groups of accessions in each country. The group of accessions in each country displayed high to very high coefficient of variation ( $>20 \%$ ) for at least five traits (Table 5). In almost all the countries, pods/plant, seeds/plant, pods with one seed and pods with two seeds had very high $(>20 \%)$ coefficient of variation (Table 5). The Gower genetic distance (Table not shown) employed to generate Figure 3 revealed the mean similarity among the 21 countries as 0.711 . Accessions from Ghana and United Kingdom were most similar with similarity coefficient of 0.887 . Countries whose accession were most distant $(0.438)$ in similarities were: Switzerland and Ghana, then Switzerland and Gambia. The variance component explained by first three PC axes amounted to 64.8\%. In Figure 3, accessions from Niger, Malawi, Madagascar and Switzerland prominently stood alone. Although there are near relationship between accessions from different countries, however accessions from each country significantly stood unique (Figure 3). There existed significant regional relationship among accessions from West Africa (Benin Republic, Burkina Faso, Guinea, Gambia, Ghana, Senegal, Mali and Cote d'Ivoire), Central Africa (DR Congo, Cameroon and Central Africa), and South Africa (Zambia and Zimbabwe). Accessions from Nigeria and Tanzania were very close in similarity (Figure 3).

Table 5. Coefficient of variation for each of the traits in the different countries

\begin{tabular}{|c|c|c|c|c|c|c|c|c|c|c|c|c|c|c|c|c|c|}
\hline Country & DOF & Wt100 & PH6 & PH8 & LL & $\mathrm{LW}$ & PL & CP6 & CP8 & PP & SP & P1S & P2S & POL & SHEL & SEL & SEW \\
\hline Nigeria & 6.76 & 14.85 & 13.52 & 33.03 & 9.00 & 7.43 & 16.06 & 15.16 & 9.42 & 26.43 & 26.08 & 28.20 & 71.26 & 22.17 & 31.56 & 13.71 & 7.96 \\
\hline \begin{tabular}{|l} 
Camero \\
on
\end{tabular} & 5.30 & 15.48 & 5.92 & 17.45 & 7.30 & 14.05 & 10.92 & 7.85 & 22.05 & 105.60 & 106.08 & 107.93 & 169.56 & 55.97 & 57.71 & 56.89 & 56.66 \\
\hline Ghana & 9.25 & 9.37 & 7.50 & 13.12 & 16.59 & 21.16 & 21.58 & 12.28 & 10.50 & 151.38 & 151.38 & 151.38 & 16.21 & 68.26 & 66.78 & 67.01 & 66.71 \\
\hline \begin{tabular}{|l|} 
Burkina \\
Faso \\
\end{tabular} & 4.28 & 15.40 & 2.22 & 4.69 & 12.06 & 8.90 & 8.40 & 16.60 & 25.66 & 151.44 & 141.42 & 164.13 & 200.00 & 118.49 & 115.58 & 115.60 & 115.48 \\
\hline Benin & 1.70 & 2.02 & 0.21 & 12.24 & 20.79 & 16.79 & 12.12 & 12.86 & 6.73 & 47.14 & 49.69 & 44.45 & 141.42 & 0.21 & 38.45 & 5.79 & 18.08 \\
\hline $\begin{array}{l}\text { Cote } \\
\text { d'Ivoire }\end{array}$ & 9.54 & 11.16 & 13.92 & 29.90 & 16.10 & 12.42 & 10.19 & 15.71 & 22.33 & 111.65 & 118.79 & 97.91 & 141.42 & 26.95 & 85.77 & 22.99 & 25.79 \\
\hline Mali & 5.61 & 13.43 & 7.27 & 5.12 & 48.97 & 35.36 & 6.26 & 24.99 & 15.38 & 72.84 & 84.62 & 58.88 & 135.40 & 18.07 & 29.04 & 6.45 & 5.80 \\
\hline $\begin{array}{l}\text { Tanzani } \\
\text { a }\end{array}$ & 4.37 & 19.43 & 36.92 & 11.42 & 25.54 & 13.98 & 10.20 & 18.00 & 13.04 & 66.67 & 71.38 & 62.14 & 158.70 & 18.77 & 21.99 & 7.96 & 4.09 \\
\hline \begin{tabular}{|l|} 
Congo \\
\end{tabular} & 17.65 & 25.04 & 2.56 & 5.00 & 15.00 & 6.63 & 6.99 & 12.86 & 10.33 & 100.66 & 119.02 & 120.00 & 120.00 & 19.29 & 26.91 & 69.28 & 67.13 \\
\hline Guinea & 11.30 & 32.29 & 6.31 & 5.55 & 3.97 & 7.29 & 8.90 & 13.86 & 30.14 & 164.17 & 164.90 & 163.31 & 173.21 & 87.55 & 86.81 & 88.35 & 87.15 \\
\hline Gambia & 12.20 & 24.81 & 11.01 & 7.12 & 7.02 & 9.78 & 18.81 & 28.30 & 19.63 & 184.54 & \begin{tabular}{ll|}
189.31 \\
\end{tabular} & 178.24 & 223.61 & 137.05 & 93.09 & 94.95 & 94.31 \\
\hline $\begin{array}{l}\text { Central } \\
\text { Africa }\end{array}$ & 10.40 & 21.93 & 7.79 & 6.37 & 15.76 & 17.07 & 15.84 & 28.02 & 24.87 & 52.15 & 55.05 & 49.11 & 120.00 & 10.13 & 15.03 & 12.72 & 21.17 \\
\hline Zambia & 11.19 & 24.66 & 12.58 & 14.07 & 7.98 & 45.89 & 5.63 & 33.47 & 28.97 & 60.74 & 60.72 & 60.91 & 63.43 & 3.55 & 9.15 & 6.49 & 13.57 \\
\hline \begin{tabular}{|l} 
Zimbab \\
we
\end{tabular} & 7.61 & 19.78 & 7.54 & 9.45 & 8.31 & 7.07 & 5.26 & 14.17 & 8.92 & 103.79 & 99.07 & 113.00 & 98.55 & 7.00 & 15.73 & 6.40 & 11.22 \\
\hline \begin{tabular}{|l|} 
Malawi \\
\end{tabular} & 5.58 & 16.59 & 9.19 & 20.37 & 17.18 & 53.07 & 10.37 & 18.00 & 18.18 & 48.82 & 51.49 & 47.26 & 91.19 & 1.77 & 7.27 & 7.99 & 3.45 \\
\hline Senegal & 10.17 & 15.96 & 10.55 & 15.05 & 6.63 & 53.81 & 10.46 & 25.34 & 20.16 & 188.07 & 186.23 & 200.00 & 158.70 & 115.51 & 126.58 & 115.48 & 115.99 \\
\hline Niger & 1.99 & 3.83 & 16.88 & 27.56 & 11.00 & 17.78 & 23.88 & 20.20 & 47.14 & 108.42 & 118.40 & 83.19 & 141.42 & 13.31 & 11.35 & 0.65 & 5.11 \\
\hline $\begin{array}{l}\text { Switzerl } \\
\text { and }\end{array}$ & 2.68 & 3.17 & 6.83 & 0.70 & 7.00 & 48.70 & 7.84 & 20.20 & 20.20 & 95.08 & 96.46 & 93.39 & 109.99 & 10.30 & 10.62 & 0.62 & 6.29 \\
\hline \begin{tabular}{|l|} 
Madaga \\
scar
\end{tabular} & 2.52 & 14.94 & 14.20 & 9.08 & 7.96 & 42.55 & 5.58 & 30.00 & 7.69 & 29.41 & 33.11 & 27.24 & 69.28 & 8.92 & 22.19 & 4.20 & 2.21 \\
\hline UK & 22.24 & 15.61 & 18.90 & 13.72 & 15.17 & 3.67 & 0.23 & 6.73 & 5.66 & 94.28 & 94.28 & 94.28 & 12.35 & 141.42 & 13.43 & 10.21 & 5.81 \\
\hline
\end{tabular}

DOF $=$ Days to flowering, Wt100 = Weight of 100 seeds, PH6 = Plant height at 6 weeks after planting, PH8 = Plant height at 8 weeks after planting, LL= Leaf length, LW=Leaf width, PL = Pod length, CP $6=$ Canopy at 6 weeks after planting, $\mathrm{CP} 8=$ Canopy at 8 weeks after planting, $\mathrm{PP}=$ Pods per plant, $\mathrm{SP}=$ Seed per plant, $\mathrm{P} 1 \mathrm{~S}=\mathrm{Pod}$ with 1 seed, $\mathrm{P} 2 \mathrm{~S}=\mathrm{Pod}$ with 2 seed, $\mathrm{POL}=$ Pod length, $\mathrm{SHEL}=$ shelling percentage, $\mathrm{SEL}=$ Seed length, $\mathrm{SEW}=$ Seed width

The total variance explained by the first two principal component axes in Figure 4 was $56.8 \%$. The interaction between the countries with the 17 quantitative traits was only feasible in quadrants 2, 3 and 4 (Figure 4). Countries with accessions with high days to flowering, shelling percentages and hundred seed weight in the second quadrant were: Cameroon, Cote d'Ivoire, Ghana, Guinea and United Kingdom. Countries with accessions with high values for vegetative, pod and seed characteristics in the third quadrant were: Central Africa, Congo, Madagascar, Nigeria, Sudan and Tanzania (Figure 4). Within the third quadrant in Figure 4, positive and significant correlation existed between petiole length, and plant height at 6 weeks after planting. However, in the fourth quadrant, seeds/plant, pods/plant and one seed per pod were positively significantly correlated and the country with the best performance for the three was Switzerland. Within the same quadrant, accessions from Malawi were most significant in the production of pods which produced two seeds. 


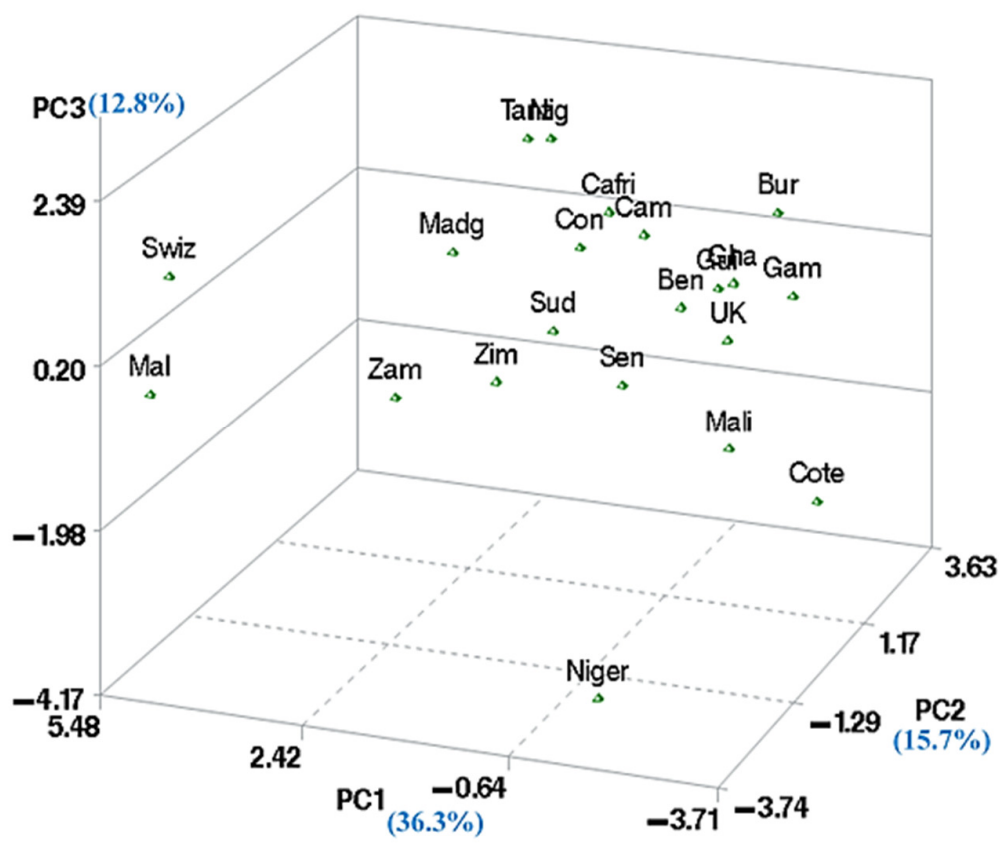

Figure 3. The display of the twenty-one countries for the different accessions they hold in a tri-dimensional figurative display

Ben - Benin Republic, Bur-Burkina faso, Cam- Cameroon, CAfri- Central Africa, Con- Congo, Cote-Cote d' Ivoire, Gam- Gambia, Gha-Ghana, Gui- Guinea, Madg-Madagascar, Mal-Malawi, Nig-Nigeria, Sen-Senegal, Sud-Sudan, Swiz-Switzerland, Tanz-Tanzania, UK-United Kingdom, Zam-Zambia and Zim - Zimbabwe

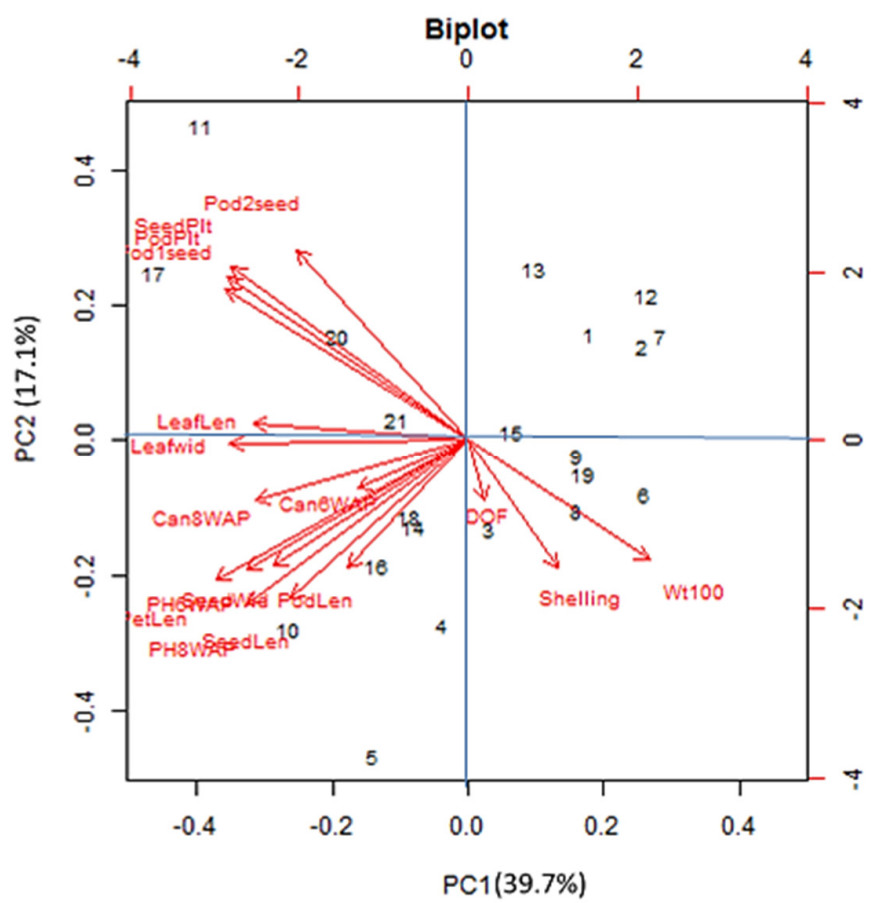

Figure 4. Biplot display of the relationship between the grouped accessions in the twenty-one countries and seventeen quantitative variables

1-Benin, 2-Burkina Faso, 3-Cameroon, 4-Central Africa, 5-Congo, 6-Cote d'Ivoire, 7-Gambia, 8-Ghana, 9-Guinea, 10-Madagscar, 11-Malawi, 12-Mali, 13-Niger, 14-Nigeria, 15-Senegal, 16-Sudan, 17-Switzerland, 18-Tanzania, 19United Kingdom, 20-Zambia and 21-Zimbabwe 


\section{Discussion}

A total of 19 morphological traits were used to identify and quantify inherent variabilities in the 61 Bambara groundnut accessions obtained from GRC, IITA, Ibadan, Nigeria. The results showed that there were wide ranges between the minimum and maximum values for the traits. This finding could provide a good resource for breeders in his selection programme. The average days of flowering from our study was higher than those obtained by Unigwe et al. (2016) in South Africa, the difference of about ten days variation could be attributed to climate and location, edaphic factors. Dimakatso (2006) described flowering in Bambara groundnut to be indeterminate. However, earliness in flowering is an economic agronomic attribute which is direct index for early maturity in fruiting crops. Moreover, the combination of earliness in maturity and high yield are key production goals in crops (Kumaga et al., 2003). In this study, TVSu1986, TVSu1844 and TVSu 1842 could be selected for early maturity because the flowered much earlier. Yield is a measure of and an indication of good agronomic and morphological characteristics Kadams and Sago (1998), in this study, TVSu1964 (Switzerland), TVSu1748 (Malawi) and TVSu1750 (Malawi) produced the highest mean seed yield. Relatively, clusters II and III grouped accessions with promising vigour and grain yield characters. For clusters II and III, intra and inter cluster selection of accessions for trait-based hybridization can lead to significant grain yield improvement. Moreover, for a short-term improvement programme, the three accessions in cluster III can be subjected to multi-environment evaluation for stability test and consistent performance among them can lead to their release to farmers for increased productivity. From the quantitative data employed for the study of the 61 accessions, significant variations exist for seed coat colours and hilum colour.

It is clear from this study that diversity in the studied genetic materials of Bambara groundnut exists in each country for genetic improvement of vigour and yield of the crop. We record quite a high diversity among the studied accessions despite that the crop is mostly an autogamy. Pasquet et al. (1999) had much earlier noted similar observation in their study on the wild and domesticated genetic resources of Vigna subterranea. Improvement programme of Bambara groundnut could therefore take into cognizance the inherent homozygous variability in individuals and through hybridization scheme to improve the diversity and create heterozygous and heterotic individuals for the economic traits. Moreover, countries which host accessions with specific characteristics are identified. We identified highly and positively associated traits e.g. pods/plant and seeds/plant, a move to select for one means the selection for the other one. Such that a targeted improvement programme for one will positively affect the other one.

Correlation is an essential statistic in plant breeding as it measures the degree of association between two or more characters (Jonah et al., 2014). In this study from the biplot graphs, significant positive correlation existed between grain yield and its components; number of pods complimented the number of seeds, i.e. the more the pods the higher the seed yield. This agrees with the result obtained by Gonne et al. (2013) who observed that a high correlation existed between pod yield and seed yield in Bambara groundnut.

According to Bonny et al. (2019) Bambara groundnut is widely cultivated in sub-Saharan Africa where it has adapted to various agro-ecosystems (steppe, savannah and forest), each of which are capable of influencing the phenotype of the indigenous Bambara genetic resources populations. The feature of very high coefficient of variation for many of the quantitative variables within each country observed in this study seem to indict the reality of the presence of considerable variability among group of accessions from the same country. Our observation indeed buttressed the remark of Eckert et al. (2010) that spatial environmental variation and ecological heterogeneity between habitats have the potential to affect the genetic structure of indigenous plant populations. With the available and exploitable genetic variability of Bambara groundnut in each country, national breeding programme can be initiated for a worthwhile improvement.

In the report by Bonny et al. (2019) Bambara groundnut populations in Cote d'Ivoire were classified into different geographical niches. To a very large extent, accessions within the same nationals and regions were similar in this study. The observed similarity at regional level seems to reveal the possibility of genetic resources exchange among member state countries through research or famers (Shengro et al., 2013). In this study, 
considerable genetic variations existed among the Bambara groundnut accessions with respect to different countries where they were sourced, the report of Valombola et al. (2019) in Namibia followed the same trend. The noticed similarities in the accessions from Nigeria and Tanzania, and between United Kingdom with Gambia and Ghana could be due to exchange of genetic resources during probable collaborative research.

\section{Conclusions}

Results obtained from this study showed that agronomic and yield parameters can be used to characterize Bambara groundnut accessions. Grouping of the various accessions was not dependent on the origin of the different accessions studied. Selection of accessions for genetic improvement can be trait-based. Accessions with higher than one seed/pod would be a useful choice in breeding for high yielding cultivars, whose production potential could support food security.

\section{Authors' Contributions}

Conceptualization: TEK, JUA and CO; Data curation TEK and CO; Formal analysis DBA; Investigation TEK, JUA, DBA and CO; Writing original draft TEK; Writing review and editing DBA, JUA and $\mathrm{CO}$.

All authors read and approved the final manuscript.

\section{Acknowledgements}

The authors appreciate the Genetic Resource Centre (GRC), International Institute of Tropical Agriculture (IITA), Ibadan, Nigeria for providing the Bambara seeds used for the study.

\section{Conflict of Interests}

The authors declare that there are no conflicts of interest related to this article.

\section{References}

Azam-Ali SN, Sesay A, Karikari SK, Massawe FJ, Aguilar-Manjarrez J, Bannayan MM, Hampson KJ (2001). Assessing the potential of the potential of an underutilized crop- crop study using Bambara groundnut. Experimental Agriculture 37:433-472. https://doi.org/10.1017/S0014479701000412

Baudoin JP, Mergeai G (2001). Grain legumes. In: Crop Production in Tropical Africa. Brussels: Directorate Generale for International Cooperation.

Bonny BS, Adjoumani K, Seka D, Koffi KG, Kouonon LC, Koffi KK, Bi ZI (2019). Agromorphological divergence among four agro-ecological populations of Bambara groundnut (Vigna subterranea (L.) Verdc.) in Côte d'Ivoire. Annals of Agricultural Sciences 64:103-111. https://doi.org/10.1016/j.aoas.2019.04.001

Dimakatso RM (2006). Evaluation of Bambara groundnut (Vigna subterranean) for yield stability and yield related characteristics. MSc Thesis, University of the Free State.

Drabo I, Sereme P, Dabire CB (1995). Bambara groundnut. In: Heller J, Engels J, Hammer K (Eds). Proceedings of the Workshop on Conservation and Improvement of Bambara Groundnut [Vigna subterranea (L.) Verdc.]; November 14-16; Harare, Zimbabwe International Plant Genetic Resources Institute, Rome, Italy. 
Eckert AJ, Heerwaarden JV, Wegrzyn JL, Nelson CD, Ross-Ibarra J, Gonzalez Martınez SC, Neale D (2010). Patterns of population structure and environmental associations to aridity across the range of loblolly pine (Pinus taeda L., Pinaceae). Genetics 185:969-982. https://doi.org/10.1534/genetics.110.115543

FAO (2020). Bambara groundnut-Traditional crop of the month. Retrieved 2020 May 25 from https://www.fao.org/traditional-crops/bambaragroundnut/en/

Goli AE (1997). Bibliographical review. In: Heller J, Begeman F, Mushonga J (Eds). Bambara Groundnut. Vigna subterranean (L.) Verdc. Proceedings of the workshop on Conservation and Improvement of Bambara Groundnut (Vigna subterranean (L.) Verdc.) 1995 conference. Zimbabwe, pp 4-10.

Gonne S, Felix-Alain W, Burgui B (2013). Assessment of twenty Bambara groundnut (Vigna subterranean (L.) Verdcourt) landraces using quantitative morphological traits. International Journal of Plant Research 3(3):39-45. https://doi.org/10.5923/j.plant.20130303.04

Gower JC (1971). A general coefficient of similarity and some of its properties. Biometrics 27:623-637. https://doi:10.2307/2528823

Heller J, Begemann F, Mushonga J (1997). Promoting the conservation and use of underutilized and neglected crops. Proceedings of the Workshop on Conservation and Improvement of Bambara Groundnut (Vigna subterranea(L.) Verdc.). Harare, Zimbabwe. Institute of Plant Genetics and Crop Plant Research, Gatersleben, Rome, Italy, pp 165.

Hillocks R, Bennett C, Mponda O (2012). Bambara nut: A review of utilization, market potential and crop improvement. African Crop Science Journal 20:1-16.

IPGRI, IITA and BAMNET (2000). Descriptors for Bambara groundnut (Vigna subterranea). International Plant Genetic Resources Institute, Rome, Italy; International Institute of Tropical Agriculture, Ibadan, Nigeria; The International Bambara Groundnut Network, Germany.

Jonah PM, Abimiku OE, Adeniji OT (2014). Multivariate analysis and character association on the growth and yield of Bambara groundnut in Mubi, Adamawa State, Nigeria. International Journal in Management and Social Science Research 3:2.

Kumaga FK, Adiku SGK, Ofori K (2003). Effect of post-flowering water stress on dry matter and yield of tropical grain legumes. International Journal of Agriculture and Biological Research 14:405-407.

Morris JB (2008). Rhynchosia minima (L.) DC. Regeneration, characterization and potential uses for natural products and flavonoids. Plant Genetic Resources Newsletter 153:15-19.

Ntundu WH, Shillah SA, Marandu WYF, Christiansen JL (2006). Morphological diversity of Bambara groundnut [ Vigna subterranea (L.) Verdc.] landraces in Tanzania. Genetic Resources and Crop Evolution 53:367-378. https://doi.org/10.1007/s10722-004-0580-2

Ntundu WH, Inga BC, Christiansen JL, Andersen SB (2004). Analysis of genetic diversity in Bambara groundnut [ Vigna subterranea $(\mathrm{L})$ Verdc] landraces using amplified fragment length polymorphism (AFLP) markers. African Journal of Biotechnology 3(4):220. http://hdl.handle.net/1807/4119

Ofori K, Kumaga F, Tonyigah A (2006). Morphological characterization and agronomic evaluation of Bambara groundnut (Vigna subterranea(L.) Verdc.) germplasm in Ghana. Plant Genetic Resources Newsletter 145:23-28. http://dx.doi.org/10.19044/esj.2018.v14n36p377

Okpuzor J, Okochi V, Ogbunugafor H, Ogbonnia S, Fagbayi T, Obidiegwu C (2009). Estimation of cholesterol level in different brands of vegetable oils. Pakistan Journal of Nutrition 8:57-62. http://dx.doi.org/10.3923/pjn.2009.57.62

Pasquet RS, Schwedes S, Gepts P (1999). Isozyme diversity in Bambara groundnut. Crop Science 39:1228-1236. https://doi.org/10.2135/cropsci1999.0011183X003900040045x

Poulter NH, Caygill JC (1980). Vegetable milk processing and rehydration characteristic of Bambara groundnut (Voandzeia subterranea (L) Thouars). Journal of the Science of Food and Agriculture 31:1158-1163. https://doi.org/10.1002/jsfa.2740311106

SAS Institute Inc (2011). SAS OnlineDoc_9.4. SAS Institute, Cary.

Shengro A, Rensburg W, Adebola P (2013). Assessment of genetic variability in Bambara groundnut (Vigna subterranea L. Verdc.) using morphological quantitative traits. Academia Journal of Agricultural Research 1:45-51. http://dx.doi.org/10.15413/ajar.2012.0129

STAR (2014). Biometrics and breeding informatics. BPGB Division, International Rice Research. 
Thottappilly G, Rossel HW (1997). Identification and characterization of viruses infecting Bambara groundnut (Vigna subterranean) in Nigeria. International Journal of Pest Management 43:177-185. https://doi.org/10.1080/096708797228645

Tweneboah CK (2000). Modern agriculture in the tropics with special reference to Ghana. Accra, Co-Wood Publishers Uguru MI, Ezeh NE (1997). Growth, nodulation and yield of Bambara groundnut (Vigna subterranea (L) Verdc.) on selected Nigerian soils. Journal of the Science of Food and Agriculture 73:377-382. https://doi.org/10.1002/(SICI)1097-0010(199703)73:3<377::AID-JSFA739>3.0.CO;2-Z

Unigwe A, Gerrano A, Adebola P (2016). Morphological variation in selected accessions of Bambara groundnut (Vigna subterranean L. Verdc) in South Africa. Journal of Agricultural Science 8:69-80. https://doi.org/5539/jas.v8n//p69

Valombola JS, Akundabweni LM, Awala SK, Hove K (2019). Agronomic and morphological diversity of Bambara groundnut (Vigna subterranean (L.) Verdc.) Accessions in North-Central Namibia. Welwitschia International Journal of Agricultural Science 1:88-99. https://doi.org/10.32642/wijas.v1i0.1369

Ward JH (1963). Hierarchical grouping to optimize an objective function. Journal of the American Statistical Association 58:236-244. https://doi.org/10.1080/01621459.1963.10500845
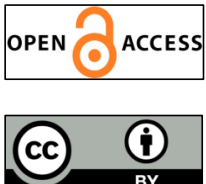

The journal offers free, immediate, and unrestricted access to peer-reviewed research and scholarly work. Users are allowed to read, download, copy, distribute, print, search, or link to the full texts of the articles, or use them for any other lawful purpose, without asking prior permission from the publisher or the author.

License - Articles published in Notulae Scientia Biologicae are Open-Access, distributed under the terms and conditions of the Creative Commons Attribution (CC BY 4.0) License.

(c) Articles by the authors; SHST, Cluj-Napoca, Romania. The journal allows the author(s) to hold the copyright/to retain publishing rights without restriction. 